Endometriosis in Norwegian Women at Interval

Sterilization

Mette Moen

University Hospital of Trondheim, Trondheim, Norway

The prevalence of endometriosis was investigated in 108 Norwegian women admitted for interval tubal sterilization. Mean age of the patients was 37 years. Endometriosis was detected in $19(18 \%)$ of the women. About one half of these had no symptoms from the disease. A positive correlation was found between a retroversion of the uterus and endometriosis. The women with endometriosis showed a longer period of uninterrupted menstrual cycles prior to the time of sterilization as compared to the controls (89 unaffected women). Sisters and mothers of patients with endometriosis displayed a higher frequency of benign gynecological disorders than the corresponding relatives of the control group. The prevalence of endometriosis appeared to be independent of following clinical parameters: onset of menarche, age at first pregnancy, number of pregnancies, previous use of hormonal contraceptives or IUD and actual age at the time of the sterilization.

\title{
Self-Help and Endometriosis
}

\section{Diane Carlton \\ Endometriosis Society, London, England}

Founded 5 years ago, the society provides emotional support and information to women suffering from endometriosis and their families. Run by a small core of trustees, there is now a network of some 60 local self-help groups, and we have been contacted by over 12,000 women. A variety of leaflets we produce on all aspects of the disease and its treatments are in great demand, both orthodox and alternative. Local and national publicity is increasing awareness of the disease. Workshops for members are run in London and bi-monthly news letters produced. With so many contacts it has been possible to provide participants for research into drug therapy, and our own survey into 800 case histories to monitor trends in treatment, rare conditions and side-effects. We also sponsor a research fund, at St. Thomas's Hospital, London.

Use of Danazol to Treat External Moderate or Severe Endometriosis in Black African Women and One Case of Uterine Adenomyosis

\author{
J.P. Brettes, Y. Djanhan, M. Anoma, S. Anonga, M. Bohoussou, A.M. Sangaret \\ Service de Gynéco-Obstétrique, CHU Cocody (Director: Prof. Sangaret),
}


Four Black African women with Pelvic endometriosis were treated with danazol 400 $\mathrm{mg} /$ day for 6-9 months. Pain relief was immediate; clinical examination showed reduction but not elimination of endometriosis sites. Side effects were secondary amenorrhea,

\section{Free Communications 384}

weight gain of $5-10 \mathrm{~kg}$, severe leg cramps. One normal birth was obtained after 8 years sterility.

One White French woman presenting uterine adenomyosis with menometrorrhagia and severe chronic anemia was given danazol 600 then $300 \mathrm{mg} /$ day. The spectacular improvement enabled hysterectomy previously envisaged to be avoided.

Comments on AFS classification of external endometriosis, the advantages of laparoscopy, the high cost of danazol, which although more efficient than progestins and estroprogestins must therefore be reserved for cases where these drugs have failed.

Clinical Presentation of Endometriosis

Jamil Sharif

Kebangsaan University, Kuala Lumpur, Malaysia

According to a study on patients at the University of Kebangsaan, Malaysia, it was noted that endometriosis did not present as described before in textbooks. The study was carried out on 50 cases. All the cases were either confirmed histologically or by direct visualisation. Among the interesting cases were a case of endometroid carcinoma and a case of endometriosis of the bladder.

Only $40 \%$ of the cases were diagnosed pre-operatively. Most of these cases were moderate to extensive and required major surgery.

Endometriosis: An Exceptional Etiology of Incidentally

Discovered Adrenal Mass

V. Durlach, C. Wallays, M. Pluot, J.B. Flament, G. Ostermann, P. Dorangeon, M. Leutenegger

Medical and Endocrine Clinic, Centre Hospitalier Universitaire de Reims, Hôpital Robert-Debré, Reims, France

A 38-year-old female with a history of hysterectomy (for a uterine fibroma) is refered for investigation of an incidentally discovered right adrenal mass. This hypoechogenic tumor had been found after abdominal ultrasonography motivated by three iterative pain episodes of the right upper quadrant. There is no clinical and biological evidence for 
hormonal adrenal hypersecretion nor primitive neoplasia. Abdominal CT scan and digitalized angiography confirm the existence of an avascular polycyclic tumor. Lombotomy is decided permitting a right adrenalectomy. Pathological analysis shows a normal adrenal gland pushed back by a lesion $2 \mathrm{~cm}$ in diameter, within a hematoma from endomeriosic origin. This etiology of incidentally discovered adrenal mass has not yet been described. Two pathophysiological hypotheses can be relevant with this ectopic localization of endomeriosis: veinous and/or lymphatic metastasis and, more likely, because of the contiguity with the peritoneal serosa and the embryological origin of the adrenal glands, celomic metaplasia. 